\title{
Towards a dynamical network view of brain ischemia and reperfusion. Part II: a post-ischemic neuronal state space
}

\author{
Donald J. DeGracia, Ph.D.* \\ Department of Physiology, Wayne State University, Detroit, MI 48201, U.S.A.
}

\begin{abstract}
The general failure of neuroprotectants in clinical trials of ischemic stroke points to the possibility of a fundamental blind spot in the current conception of ischemic brain injury, the "ischemic cascade". This is the second in a series of four papers whose purpose is to work towards a revision of the concept of brain ischemia by applying network concepts to develop a bistable model of brain ischemia. We here build the bistable network model of brain ischemia. The central concept is that of a post-ischemic state space. Ischemia, as a quantitative perturbation, is envisioned to push the brain through a series of four phenotypes as a function of the amount of ischemia: the homeostatic, preconditioned, delayed neuronal death and necrotic phenotypes. The phenotypes are meta-stable attractors in the landscape of the post-ischemic state space. The sequence of the phenotypes derives from the mutual antagonism between damage mechanisms and stress responses, each conceived as aggregate ensemble variables. The competition between damage mechanisms and stress responses is posited to have the form of a bistability. Application of bistability to brain ischemia is grounded in the incontrovertible fact that post-ischemic neurons face the mutually exclusive decision to either live or die.
\end{abstract}

\section{Keywords}

Bistability; global cerebral ischemia; neuroprotection; preconditioning; post-ischemic state space

\section{Why "towards" a dynamical network view of brain ischemia and reperfusion?}

\begin{abstract}
We titled this series of papers "Towards..." for a good reason: we do not here present a formal network model of brain ischemia and reperfusion. A formal network model would consist of a set of equations, the answer to which is the state space. Instead, we discuss considerations that can move us toward constructing such a formal network. We in fact work backwards, discussing what the state space of the post-ischemic brain should look like based on our empirical understanding of outcomes after brain ischemia. We then go under the hood of this hypothetical post-ischemic state space and show how it can be derived from a particular circuit motif, the bistable motif. Bistability results whenever a system faces two mutually exclusive states (Chatterjee et al 2008). Post-ischemic cells indeed face a mutually exclusive decision: live or die.
\end{abstract}

\footnotetext{
(C) 2010 SFES

*Correspondence should be sent to: Donald J. DeGracia, Ph.D., Department of Physiology, Wayne State University, 4116 Scott Hall, 540 East Canfield Ave., Detroit, MI 48201, U.S.A.; Phone 313-577-6745; Fax 313-577-5494; ddegraci@ med.wayne.edu.

Conflict of interest

None
} 
The net result of our present effort is that we end up with a faux network model of brain ischemia. The model is faux because there are no network equations behind it. However, as we shall see, a post-ischemic state space is in fact constructed from empirical considerations. We will discuss the additional hurdles required to convert this empirical state space to actual network equations. The present work is a necessary step towards discovering such equations by laying out the rationale and justifications for constructing the state space. The hope is that by airing these considerations in the literature, it will facilitate the discovery of the state space equations. So while the resulting model is "faux", the discussions leading up to it are not, and we shall see (mainly in the $3^{\text {rd }}$ paper) that the resulting post-ischemic state space proves surprisingly useful even at its present stage of development. We begin by providing an overview of the ideas and then filling in the details.

\section{Overview}

There are three steps we follow, the cumulative result of which is to effectively outline a bistable model of brain ischemia. Step one consists of describing the post-ischemic state space as a means to represent the phenotypes cells acquire after experiencing specific amounts of ischemia. The second step is to ask how the state space landscape and associated phenotypes are generated. This will be seen to be due to the inherent competition of the damage mechanisms and the stress response, each considered as aggregate or ensemble variables. The third step is to show how a state space derived from the competition between the damage mechanisms and stress responses is in fact an example of a bistable system. These three steps constitute the task of the present paper. However, additional development of the bistable model of brain ischemia continues throughout the $3^{\text {rd }}$ and $4^{\text {th }}$ papers, elaborating on the basic framework presented below.

\section{The post-ischemic state space represents ischemia-induced phenotypes}

The essence of a network view of brain ischemia is to recognize that increasing amounts of ischemia will move the brain through distinct phenotypes. While ischemia is clearly a perturbation to the brain, we here think of this perturbation as analogous to, in some sense, a force. While we do not literally mean "force" in the technical physics sense, we need to metaphorically think of ischemia as "pushing" the configuration (or state vector) of a cell into other phenotypes. The strength of this "push" is directly proportional to the amount of ischemia, $I$, as conceptualized along the lines presented in the $1^{\text {st }}$ paper.

For reasons also discussed in the $1^{\text {st }}$ paper we consider only complete global ischemia (CGI) and take its intensity (e.g. the amount of ischemia) to be synonymous with its duration. In this and the subsequent two sections we build the post-ischemic state space, a landscape consisting of topological features (hills and valleys) that represent the possible pheno-types induced in cells by increasing amounts of ischemia, $I$.

Our point of departure is the following: Peter Lipton's quote in the $1^{\text {st }}$ paper talked about $a$ threshold of ischemia, as does Wieloch's sandwich model, but our present understanding of brain ischemia and reperfusion points to at least three important ischemic thresholds, three specific amounts of ischemia, which, upon passing, give rise to three distinct phenotypes in the post-ischemic brain.

\section{The brain's normal steady state (S)}

The first step in describing post-ischemic phenotypes is to define our baseline, which is the normal brain that has not experienced ischemia. To this end, using cellular differentiation as our case study serves a dual purpose. Below in a subsequent section, it will provide a network model of bistability we can emulate and apply to ischemia. Here however it 
provides for us a model of the normal state of brain cells, which we can utilize as our baseline. Each terminally differentiated cell type in the brain got there by differentiation. That is, each cell type in the mature brain occupies its own stable attractor in the gene network state space of the organism. The attractor of each terminally differentiated cell type exists on a landscape containing all the cell types coded by that genome; these are the epigenetic landscapes first described by CH Waddington (1957). This attractor provides our baseline and we call this baseline the steady-state, S, of the terminally differentiated cell. S is that point in the organism's gene network state space that corresponds to the configuration (i.e. pattern of gene expression) of the terminally differentiated cell type.

An advantage of focusing only on CGI is that, unlike focal ischemia, the main target of global ischemia is the neuron (at least over a meaningful range of ischemic intensities). This allows us to narrow our focus specifically to neurons. However, the following logic would be expected to be applicable to the glia, vascular and immunological cells that are also altered following focal ischemia (discussed in the $4^{\text {th }}$ paper). But for our present purposes, $\mathrm{S}$ is taken to be that point in the genetic state space of an organism that represents the attractor of terminally differentiated neurons. After presenting the state space, we discuss the issue of neuron subtypes.

\section{The homeostatic state $(\mathrm{H})$}

Now, while $\mathrm{S}$ is the lowest (e.g. most stable) point in the attractor of the neuron phenotype, there is still the rest of the valley. Technically, the valley surrounding an attractor point is called the basin of attraction. A basin of attraction is defined as the collection of points in the state space having the property that, if the system visits a basin point, it will inevitably settle back to the attractor point (Huang 2009). For our purposes here, we consider the basin of attraction surrounding $S$ to represent states that are homeostatic responses of the neuron to sub-pathological changes in blood flow. We thus term the basin of attraction around $\mathrm{S}$ the homeostatic state, $\mathrm{H}$ (Figure 1). Responses of neurons in $\mathrm{H}$ fall into two classes: (1) points close to $S$ represent normal physiological responses (green region, Figure 1), and (2) points farther away from $\mathrm{S}$ represent more extreme responses that are supra-physiological but could be considered sub-pathological (red region, Figure 1). We now explain the rationale of this distinction.

The first distinction arises because of the relationship between blood flow and neuronal function. It is well-known that neuron activity and local cerebral blood flow (CBF) are intimately related in the process of flow-metabolism coupling (Iadecola and Nedergaard 2007). Therefore, there is a nontrivial range of blood flows which underlie flow-metabolism coupling that will produce acute homeostatic changes in neurons. Such changes underlie normal neuron function including conduction, memory, etc. Neuronal responses in the regime indicated by the green region of Figure 1 will include genetic changes underlying normal neuronal plasticity. Technically, what this implies is that the point $\mathrm{S}$ is not really a fixed point, but will alter as a function of neuronal plasticity and thus form a kind of probabilistic "cloud" (Huang, 2009). However, this is a subtlety not essential to our arguments and is mentioned only for the sake of completeness. For the purpose of building the post-ischemic state space, we may make the approximation that $S$ is a fixed point in the organisms' genetic state space. Therefore, in terms of acute responses to changes in local $\mathrm{CBF}$, we can take the points close to $\mathrm{S}$ to represent normal physiological responses of a neuron.

However, a neuron can experience homeostatic disruptions that fall in a gray area between normal and pathological. Application of a small amount of CGI to a neuron falls in this gray area. We know that a very short duration of CGI (e.g. $<1 \mathrm{~min}$ ) is not lethal to neurons. There will however be a momentarily displacement of neurons out of their normal energetic 
steady-state (Siesjö and Wieloch 1985). ATP levels will drop, but not to zero, nonetheless triggering corresponding acute energy conservation processes in the neuron. These are very short-lived effects, lasting only minutes after removal of a very brief CGI perturbation, after which time the neuron returns to its normal steady state, S.

In fact, the state we are describing here is what Kirino (2002) called "immediate preconditioning". This form of preconditioning is more predominant in the heart, but it is also present in the brain. The essence of the supra-physiological range of the $\mathrm{H}$ state is the activation of acute homeostatic responses that are outside the neuron's normal response range, but that do not involve genetic reprogramming of the neuron into a stress response phenotype in response to the decrement in blood flow. Again, such supra-physiological responses occur when $I<1 \mathrm{~min}$, but greater than the normal range of physiological local CBF variation.

With respect to the state space, this implies that there is some range around $\mathrm{S}$ in the basin that is relatively flat and contains the response of a neuron to normal homeostatic variation (again, flow-metabolism coupling that would underlie normal neuronal plasticity). However, the second part of the basin where the supra-physiologic homeostatic disruption is represented is expected to have a steep slope. This slope would reflect the relatively intense neuronal responses to a disruption such as brief CGI, which in turn would induce a rapid return back to steady-state, $\mathrm{S}$, after the disruption. In the case of immediate preconditioning, neurons reset to $S$ within < 15 min after the brief CGI (Kirino, 2002). These ideas are illustrated in Figure 1.

\section{A "time out" on what the state space represents}

Figure 1 is our first example of the marble moving on the post-ischemic state space landscape. We would like to clarify what this represents as it will be a recurring theme throughout the rest of the presentation. The moving marble is a visual device for depicting changes in the state vector of the neuron induced by the "force" of ischemia. In the $1^{\text {st }}$ paper, we used examples where a point in the state space corresponded to a specific pattern of gene expression. However, in the post-ischemic state space each point represents the many responses of a neuron to the applied ischemia.

As stated in the $1^{\text {st }}$ paper these responses fall into two classes: damage mechanisms and stress responses. The reader may wonder how many damage mechanisms and many stress responses can be represented merely as a point. As explained in the $1^{\text {st }}$ paper, state spaces are multi-dimensional. A point in the state space can have any arbitrary $n$ components, each representing the state of one of the $n$ nodes of the network. Thus, in the post-ischemic state space, each point represents all ischemia-induced damage mechanisms and stress responses, the entirety of which are taken to be the phenotype of the post-ischemic neuron. At this point, we simply introduce this key idea. We will continue to develop this notion into the $3^{\text {rd }}$ paper and ask the reader's patience as the concept is developed. At this point in the discussion, the key idea is that movement of the state vector (marble) through the state space represents changes in the phenotype of the neuron. On a certain level, this idea is not at all unfamiliar. Mendel (1866) worked in terms of pheno-types. The only difference between the classical Mendelian view and the network view is that the network view incorporates the idea of dynamic changes of phenotypes in real time and applies the concept to individual cells.

\section{The preconditioned phenotype (P)}

A somewhat greater amount of CGI ( 2-5 min) is still not lethal to neurons, but will push them over the first ischemic threshold, which we term the preconditioning threshold, $T_{P}$. 
Theoretically, $T_{P}$ is a specific amount of ischemia, a specific value of $I$. Exposure to amounts of ischemia equal to or greater than $T_{P}$ puts neurons into a phenotype identifiably different from those in $\mathrm{S}$ or $\mathrm{H}$. Neurons exposed to amounts of ischemia $I>T_{P}$ (but below a lethal threshold discussed below), can now withstand a subsequent lethal insult which neurons in S cannot withstand. This phenotype is what Kirino (2002) referred to as "delayed preconditioning" but we will refer to simply as preconditioning. While the field in general focuses on preconditioning as a form of neuroprotection, we here use the fact only as a marker that passing $T_{P}$ has put the neurons into a new phenotype. This phenotype lasts much longer than changes induced by $\mathrm{H}$ quantities of ischemia, but it is still completely reversible, persisting for several days before the neurons revert back to their normal steady-state, $\mathrm{S}$ (Kirino 2002). The preconditioned state consists not only of acute compensatory changes as found in $\mathrm{H}$, but also longer term changes in gene expression and protein synthesis that are responsible for its time course of expression (Kirino 2002). Thus, preconditioning is a form of temporary genetic reprogramming into a stress response phenotype. It is the genetic reprogramming into a stress response phenotype that distinguishes $\mathrm{P}$ phenotypes from $\mathrm{H}$ phenotypes, the latter of which do not so genetically reprogram.

From the point of view of the neuronal state space, we envision that the preconditioned phenotype $\mathrm{P}$ occupies a plateau of responses that, on one hand are outside the normal basin of attraction $(\mathrm{H})$ of the neurons, but on the other hand extend the basin of attraction (Figure 2). The slope of this plateau is such that it does not form a permanently stable attractor valley, but instead has a very gently rising slope oriented such that the state vector will fall back to $S$. The slope in this case is intended to reflect the much slower kinetics of onset and shut-off of the preconditioned phenotype. However, since the plateau is sloped towards S, the neurons will eventually and inevitably fall back to $S$ (illustrated by the trajectory of the marble in Figure 2). At some upper limit of CGI (a threshold we call $T_{D}$, discussed in the next section), the neurons will no longer experience preconditioning as an outcome, setting the extent of this phenotype on the scale of CGI. In terms of state space, we envision the upper amount of ischemia to set a wall (a "quasi-potential" barrier) in the state space, an issue discussed further ahead. Thus, $\mathrm{P}$ is a meta-stable, reversible phenotypic alteration of the post-ischemic neuron. We are mute for the moment about the genesis of this and the subsequent phenotypes, returning to that consideration after the post-ischemic neuronal state space has been described.

\section{The delayed neuronal death phenotype (D)}

If we continue to increase the intensity of CGI past that required for preconditioning (e.g. to $\sim 10 \mathrm{~min}$ ), the second ischemic threshold is passed and this causes the delayed death of specific neuron populations, the selectively vulnerable neurons, yet spares resistant neurons (Kirino 2000). We therefore call this threshold amount of ischemia $T_{D}$, the threshold of delayed neuronal death (DND). Critically however, it is less the issue that the cell death is delayed than that the cell death occurs after the ischemic perturbation has been lifted. That is, the critical feature of passing $T_{D}$ is that the cell death will occur during reperfusion. Before discussing the associated state space, let us describe the final post-ischemic phenotype.

(A brief note on abbreviations: DND is delayed neuronal death and refers to the phenomenological death of neurons during reperfusion; $\mathrm{D}$ is the phenotype in the state space; $T_{D}$ is the threshold amount of ischemia required to induce DND in a real brain or to move the configuration to the $\mathrm{D}$ phenotype in state space). 


\section{The necrotic phenotype (N)}

Finally, if the intensity of CGI is further increased (say to $\sim 30 \mathrm{~min}$ ), we pass a third ischemic threshold and move into a domain in which the ischemia is so intense that the neurons die acutely during the ischemia by necrosis (Siesjö and Smith 1991). The minimum amount of ischemia to trigger cell death during the ischemia itself is the necrotic threshold, $T_{N}$, and the phenotype is $\mathrm{N}$, the necrotic phenotype.

\section{Brief summary of post-ischemic phenotypes}

Before continuing, we briefly summarize. A very small amount of CGI will displace a neuron from $\mathrm{S}$ and cause it to enter the homeostatic state $\mathrm{H}$, which is a very rapid and short lived response. Amounts of ischemia from $T_{P}$ to $<T_{D}$ will induce preconditioning in a neuron. Between $T_{D}$ and $T_{N}$, some neurons, selectively vulnerable neurons, will die during reperfusion (i.e. after the ischemia) and therefore in a delayed fashion with respect to the ischemic perturbation. Finally, if neurons experience amounts of ischemia $>T_{N}$, they will die by necrosis during the ischemia.

Thereby we can see the critical role that the concept of the amount of ischemia, $I$, plays in the present thinking. It is the dependent variable of the model.

\section{The post-ischemic state space and cell death}

We now discuss adding cell death as a feature of the post-ischemic state space. To do so, we introduce two critical features into the post-ischemic state space.

First, stated simply, cell death can be envisioned as an attractor in the state space. We do not here attempt a detailed justification as this has been provided by others (Huang and Ingber 2000). However, by way of a brief justification, we note that apoptosis is a genetic program. As such, it is a specific pattern of gene expression and therefore occupies an attractor in the genetic state space of an organism. Having said this, we want to strongly emphasize that this does not mean that we are saying post-ischemic neurons die by apoptosis, although many workers possess this viewpoint. In the present work we are completely agnostic about the mechanisms of cell death from the point of view of individual molecular pathways.

However, that apoptosis is a genetic program is incontrovertible, and provides the most obvious and ready example of how cell death can be a phenotypic outcome of a genetic program and therefore an attractor in an organism's genetic state space. Thus, we can depict cell death in the post-ischemic state space by the addition of a second attractor to the state space (Figure 3). We will call this point in the state space the "cell death" (CD) attractor to be as general as possible, and to not insinuate a commitment to any specific molecular pathway of cell death.

The second feature we must introduce is a specific amount of ischemia that, for technical reasons to be explained ahead, we call $I_{S}$, the amount of ischemia at the separatrix. $I_{S}$ is the "watershed" point on the state space curve. At $I_{S}$, the cell is at the tipping point between survival and death. As such it serves a function similar to Lipton's and Wieloch's concept of a cell death threshold (discussed in the $1^{\text {st }}$ paper). However, for reasons to be elaborated as we proceed, the concept of $I_{S}$ is fundamentally a more systematic concept as it is a distinct property of the post-ischemic state space. $I_{S}$ functions such that the sign of the slope of the state space curve reverses after $I_{S}$.

With addition of the attractor $\mathrm{CD}$ and the point $I_{S}$, we can now build the $\mathrm{D}$ and $\mathrm{N}$ phenotypes into the post-ischemic state space. 
As a first approximation, we model the $\mathrm{D}$ phenotype to occupy a broad, gently sloped plateau, similar to P. Again the slope is used to reflect kinetic considerations, e.g. that cell death or recovery is delayed. The D plateau however, extends over a range of $I$ that includes $I_{S}$. Thus, the slope of the state space in D will reverse at $I_{S}$. If the amount of ischemia is to the left of $I_{S}$, then the neuron will gradually fall back to $\mathrm{S}$ and survive the insult. This allows us to account for ischemia-resistant neurons. If the amount of ischemia puts the neuron to the right of $I_{S}$, the cell will gradually but inevitably fall to the cell death attractor, CD. Thus we account for ischemia-vulnerable neurons.

Finally, if a neuron experiences an amount of ischemia $I>T_{N}$ then the state space configuration moves during the ischemia on a trajectory from $\mathrm{S}$ directly to $\mathrm{CD}$, and acute cell death results during the ischemia.

\section{How to use the post-ischemic state space}

The whole of our expected post-ischemic state space is summarized in Figure 3 which depicts a two dimensional cross-section (e.g. a curve) through the state space landscape (the 3D state space is described later). The way the state space is intended to be understood and utilized is the following. The neuronal state vector is initially at $S$, the normal steady-state. Application of some specific amount of ischemia, $I$, will displace the state vector to the point on the state space corresponding to that amount of ischemia. Two arbitrary amounts of ischemia are shown in Figure 3. Applying an amount of ischemia $I_{l}$ to the point $\mathrm{S}$ "pushes" the state vector from $S$ to the point depicted by marble $M_{1} . M_{1}$ is in the region of delayed neuronal death, D, to the left of $I_{S}$ (e.g. $\left.I_{1}<I_{S}\right)$. After the state vector is so moved to that point on D, and the ischemia has ended, the state vector (marble) will then move ("roll") leftward along the state space surface until it settles back to the point $S$. Hence $\mathrm{M}_{1}$ represents a neuron that survives the ischemia. $\mathrm{M}_{2}$ on the other hand is "pushed" by a greater amount of ischemia $I_{2}$, to the right of $I_{S}$ in region D (e.g. $I_{2}>I_{S}$ ). Thereafter, marble $\mathrm{M}_{2}$ will "roll" across the state space landscape to attractor $\mathrm{CD}$ and die. $\mathrm{M}$ therefore represents the state vector of an ischemic- 2 vulnerable neuron. The rate at which a state vector (marble) moves is reflected by the slope of the surface over which it moves.

One can readily see the obvious analogy between the post-ischemic state space and (frictionless) gravity. Ischemia, as a "force", imparts "energy" to the state vector (represented by the marble), "kicking" it uphill on the state space landscape. Then, analogous to releasing this energy, the state vector (marble) "rolls" back downhill to the lowest point, either S or CD. While this is a useful metaphor, it is technically inaccurate because the heights are quasi-potentials, not a real potential like gravity (Huang 2009). But the height is nonetheless a measure of the stability of the state vector and the analogy is accurate in that regard.

What this model physically represents is how ischemia elicits responses, phenotypes, in the post-ischemic neuron. But these responses are unstable states for the neuron. To eliminate the instability in its phenotype, the neuron will either return back to its stable steady-state (S) or it will die (CD). The choice depends on the specific magnitude of ischemia, $I$, it has experienced.

\section{The post-ischemic state space: issues and features}

While the above first-pass at a post-ischemic state space offers many advantages (which are discussed in the $3^{\text {rd }}$ paper), there are also obvious problematic issues we here briefly discuss. We consider: (1) neuronal subtypes, (2) the relationship between the P and D phenotypes, (3) cell death during either ischemia or during reperfusion, and (4) the question of reperfusion-induced damage. 


\section{Neuronal Subtypes}

We built the concept of the state space as if it belongs to that of a generic neuron. However, this is inaccurate and the issue requires clarification. One may think of a "Valley of the Neurons" in an organism's genetic state space (Figure 4A). This valley will have within it many smaller local valleys, each being the attractor of a specific neuron type. We know different subtypes of neurons have different relative vulnerabilities to ischemia (Kirino 2000). For example, hippocampal CA1 neurons are most vulnerable to DND, and brainstem neurons require considerably more ischemia to induce a comparable response. Therefore, the post-ischemic state space shown in Figure 3 should be thought of as a generic template for which the specific state spaces of individual neuron populations in individual organisms will need to be constructed. This is illustrated in Figure 4B showing a family of state spaces corresponding to different neurons in an organism after exposure to the same scale of CGI. Each neuron sub-type would be expected to display its own characteristic value of $I_{S}$.

\section{The relationship between $\mathbf{P}$ and $\mathrm{D}$}

Next we consider the distinction between the $\mathrm{P}$ and $\mathrm{D}$ ranges of phenotypes. The main reason we distinguish $\mathrm{P}$ and $\mathrm{D}$ as separate plateaus is that no cell death occurs on the $\mathrm{P}$ plateau (e.g. below the $T_{D}$ amount of ischemia), whereas cell death is imminent on the $\mathrm{D}$ plateau. This line of thinking raises important questions that have not generally been addressed. Preconditioning is generally not considered in relation to neurons that survive amounts of ischemia lethal to other neuron types (e.g. the selective vulnerability of CA1 after 10 min global ischemia vs. cerebral cortical or dentate granule cells that survive this insult). Our model makes conceivable the possibility that an ischemia-resistant neuron to the left of $I_{S}$ in the D range (e.g. green range of D in Figure 3) is in fact in a preconditioned state. Such logic would apply, for example, to a dentate granule cell that will survive 10 min of global ischemia in a rat. This would then mean there is not a wall or boundary between the $\mathrm{P}$ and $\mathrm{D}$ states and any phenotype in the range $T_{P} \leq I \leq I_{S}$ would be in some gradation of preconditioned states. The two possibilities are shown superimposed in Figure 5. This question is important because the form of the P-D boundary speaks to the form of the equations required to generate the state space (discussed ahead).

If there is a P-D boundary, it may represent a probabilistic transition between recovery and cell death. The $P$ range could be defined where the probability of cell death is strictly 0 . In the D range when $I>I_{S}$, the probability of cell death could be defined as strictly 1.0. Then, the range $T_{D} \leq I \leq I_{S}$, constituting the P-D boundary, may represent a range of increasing probability of cell death following some probability function $P(I)$.

While we are in no position to offer firm answers to these questions here, the utility of the bistable model is in raising these questions in the first place, questions with clear empirical implications.

\section{Cell death during either ischemia or during reperfusion}

A critical feature of the state space is that it provides a natural classification of post-ischemic cell death. Cell death occurs either during ischemia or after the ischemia has been lifted. While this is well-known, the ideas presented here formalize this distinction, providing a systematic means by which to classify ischemia-induced cell death, a topic explored in the $3^{\text {rd }}$ paper of the series. In the terminology used here, the necrotic phenotype $\mathrm{N}$ means cell death during ischemia. The $\mathrm{D}$ phenotype refers to any death occurring after the ischemia has been lifted, or during reperfusion. 


\section{Reperfusion-induced damage}

While the idea of "after ischemia" is in practice the same as "during reper-fusion", the distinction in the present context is important. In the scope of the above ideas it is the amount of ischemia, $I$, and not reperfusion that is the driving force of the system and the dependent variable of the model. It is well-established that additional damage mechanisms are initiated specifically in response to reperfusion (Safar 1986). However, we do not here distinguish between ischemia-induced and reperfusion-induced damage mechanisms. These are lumped together generically as "damage mechanisms".

While this may appear to be a limit of the current way of thinking, the justification for doing so is the following. Reperfusion-induced damage mechanisms are not independent of events initiated during ischemia. They are in fact a consequence of them. Consider for example lipid peroxidation, a reperfusion-specific phenomenon (Chan 1994). The degree of lipid peroxidation during reperfusion will be a function of the amount of suitable substrates produced during ischemia by lipolysis and delocalization of heavy metals (Traystman et al 1991; White et al 2000). Such substrates are not significantly present in a normal brain undergoing perfusion. Thus, it is not so much that reperfusion specifically induces its own forms of damage. It is more the case that certain forms of ischemic damage are latent during ischemia and only manifest during reperfusion due to the additional substrates provided by the return of blood. Or in other words, damage during reperfusion is just an extended form of damage during ischemia. In fact, the same logic holds for stress responses as well. Most stress responses manifest during reperfusion, but in all cases the driving force is the net set of changes induced by ischemia. Therefore, given the causal links between ischemiainitiated events and subsequent events during reperfusion, we feel justified to generically lump both forms of cell damage together. This consideration then leads us to the next major topic: the genesis of the phenotypes from the competition between damage mechanisms and stress responses.

\section{Damage mechanisms and stress responses are driven by the amount of ischemia}

Here we discuss the competition, the mutual antagonism, between the stress responses and the damage mechanisms. The shape of the post-ischemic state space landscape, that is, the series of phenotypes $\mathrm{H}, \mathrm{P}, \mathrm{D}$ and $\mathrm{N}$ will be seen to naturally follow from this competition. First we approach this competition from an intuitive perspective and then briefly provide a more systematic analysis. The present discussion sets the stage for us to consider bistability as the underlying circuit motif of the post-ischemic state space in the next section.

We note that we now begin to transition to a view in which the damage mechanisms and stress responses are seen to be variables in their own right, variables that change as a function of $I$, the amount of ischemia. To this end, we define $D M$ as the total effective damage produced by all damage mechanism in response to some amount of ischemia, $I$. We define $S R$ as the total effective stress response capacity all of combined stress responses induced in a neuron by a given $I$. By defining $D M$ and $S R$ in terms of totals, we move towards viewing the aggregate or net effects of ischemia-induced damaging or protective responses. In the $3^{\text {rd }}$ paper we further formalize $D M$ and $S R$. At this point we work with the qualitative definitions just provided.

\section{Intuitive considerations}

Let us first contemplate the $D M / S R$ competition intuitively. It is clear that the intensities of both $D M$ and $S R$ will be a function of the amount of ischemia, $I$. Both should increases as $I$ increases. However, the increase is obviously not unbounded. When $I \geq T_{N}$, cell death 
ensues, ending the competition in favor of total damage, $D M$. In contrast, for a significant range in the state space, for all values of $I<I_{S}$, the stress responses, $S R$, win out over the total damage, $D M$, and the cell survives. These considerations lead us to hypothesize that the genesis of the thresholds reflects significant changes, milestone events, in the relative relationship between $D M$ and $S R$. A likely scenario is as follows (see in conjunction with Figure 6). Note that we now also begin to speak of the state space ranges H, P, D and N as synonymous with specific ranges of amounts of ischemia.

In $\mathrm{H}$, preexisting stress responses (e.g. not requiring transcription and translation) are rapidly activated, but $I$ is low, therefore the total damage is relatively weak. That is, in $\mathrm{H}, S R$ > $D M$. It seems most probable that what sets the $T_{P}$ threshold is the intracellular concentration of ATP $\left([\mathrm{ATP}]_{\mathrm{i}}\right)$. In the $\mathrm{H}$ range, $[\mathrm{ATP}]_{\mathrm{i}}$ does not reach zero. $[\mathrm{ATP}]_{\mathrm{i}}$ begins to approach zero after CGI in a time frame consistent with preconditioning amounts of ischemia (e.g. $\sim 2$ $\min$ in the gerbil, Mrsulja et al 1986). After $[\mathrm{ATP}]_{\mathrm{i}}=0$, we expect a precipitous rise in total damage, $D M$, as well as a corresponding increase in total stress response capacity, $S R$, to cope. After $T_{P}$, the increase in $S R$ is reflected in the genetic reprogramming of neurons to the $\mathrm{P}$ phenotype. However, cell death never occurs during $\mathrm{P}$ which leads to the obvious conclusion that $S R>D M$ through the entire range of $\mathrm{P}$. It continues that $S R>D M$ upon entering $\mathrm{D}$, and the issue of whether the P-D boundary is a continuum or makes a discreet break at $T_{D}$ was discussed above (Figure 5).

Upon entering $\mathrm{D}$, we encounter two new factors. The first involves saturation of the stress responses. As $I$ increases through $\mathrm{D}$, the total damage, $D M$, will continue to increase. However, we expect that total stress response capacity, $S R$, will begin to level off. We expect $S R$ to level off because of saturation. This is a key point. Stress responses are, in general, enzyme-mediated. As with any enzyme system, stress responses are subject to saturation. At some value of $I$, the amount of damage produced will definitively exceed the capacity of the induced stress responses to process the damage, hence saturation. On the other hand, many of the $D M$ are not enzyme-mediated but are non-physiological chemical reactions induced by toxic products formed during ischemia. As such, they are limited only by substrate availability (e.g. lipid peroxidation, for example, is not enzyme-mediated, and will continue as long as substrates are available). Thus, we do not expect saturation to be a general property of $D M$.

Second, recall that $S R$ and $D M$ are mutually inhibitory. Since $D M$ increases across the D range, but $S R$ saturates, this would set up a situation where the inhibition of $S R$ by $D M$ has a greater and greater effect. That is, we expect an acceleration of the inhibition of $S R$ by $D M$ through D. This will cause a decrease in the maximum (saturated) amount of $S R$ and cause the dip in $S R$ shown at the top end of the D range (green curve, Figure 6).

In short, once $T_{D}$ is passed, $S R$ will initially level off due to saturation, and then decrease because of the accelerated inhibition by $D M$. At some point in D therefore, $D M$ will become greater than $S R$. The amount of ischemia, $I$, after which $D M>S R$ is to the right of $I_{S}$. Or said slightly differently, $I_{S}$ is that amount of ischemia where $S R=D M$ (Figure 6, dashed black line at $I_{S}$ ). Defining $I_{S}$ as the amount of ischemia at which $S R=D M$ in fact provides a formal rationalization of why $I_{S}$ is the tipping point between cell survival or cell death.

Finally, when $I>T_{N}$, the $S R$ are completely overwhelmed, the $D M$ completely dominate, and the cell dies acutely during the ischemia, giving rise to the $\mathrm{N}$ phenotype.

Figure 6 illustrates the above points in two ways. First, changes in $D M$ and $S R$ are depicted by scales measuring their relative intensities, metaphorically depicted as weight. Second, changes of $D M$ and $S R$ are displayed in a more precise fashion as hypothetical curves that change as a function of the amount of ischemia, $I$. The curves can be designated as functions 
$f$ and $g$ such that $D M=f(I)$, and $S R=g(I)$. The curves $f$ and $g$ in Figure 6 are hypothetical, but nonetheless educated guesses of the relative intensities of $D M$ and $S R$ as functions of $I$, based on the considerations discussed above. While hypothetical, these curves in fact are quite important. In principle, the post-ischemic state space can be derived using the equations that would generate the curves $f$ and $g$ as input functions. If real empirical curves of $f$ and $g$ can be measured, a formal network model will be possible. We return to this key issue ahead when discussing mapping the curves $f$ and $g$ to a bistable state space.

\section{Kinetics verses thermodynamics}

The above is intuitive and informative as it goes, but there is a more systematic way to approach the issue of the relative intensities of $S R$ and $D M$ as a function of $I$. This involves the general viewpoint that any chemical reaction can be controlled either by kinetic or thermodynamic factors (Sykes 1986). Kinetic factors predominate if the rate of a reaction is very fast: fast reactions occur when the energy of activation is low. If time allows, the most stable reaction products will form, allowing thermodynamic equilibrium to dominate the system. The most stable reaction products are those with the lowest energy (or highest entropy), although getting to them may require passing a higher energy of activation. The reader will recall that the fastest reaction product is not always the most thermodynamically stable. Both kinetic and thermodynamic forms of control factor importantly in the sequence $\mathrm{H}, \mathrm{P}, \mathrm{D}$ and N. Kinetic control dominates the beginning and end of the sequence, and thermodynamic control dominates the middle of the sequence.

Events in both $\mathrm{H}$ and $\mathrm{N}$ occur because very fast reaction kinetics are involved. $\mathrm{In} \mathrm{H}$, rapid execution of pre-existing homeostatic mechanisms allows the cell to quickly re-enter $\mathrm{S}$ upon lifting the slight ischemic perturbation (e.g. Figure 1). In N, the cell proceeds to the attractor $\mathrm{CD}$ because, at high amounts of ischemia, the rate of damage massively overwhelms the rate at which the cell can cope, e.g. the rate of stress response onset. Thus, in $\mathrm{H}$ and $\mathrm{N}$, the relative intensities of the $D M$ and $S R$ are set by kinetic factors.

In $\mathrm{P}$ and $\mathrm{D}$, however, events happen over relatively much longer time periods. $\mathrm{P}$ takes time both to onset and to decay. This is clearly due to the requisite changes in transcription and translation leading to the $\mathrm{P}$ phenotype, and the eventual decay back to the $\mathrm{S}$ phenotype. Similarly in D, it takes time for the cell death to occur; hence we call it "delayed". Whatever the specific constellation of events underlying DND, we can infer, on the basis of the relative time frame involved, that these involve some type of steady-state equilibrium between $D M$ and $S R$. It cannot be a true thermodynamic equilibrium because the system under consideration, the ischemic brain, is not a closed system; hence a steady-state is expected to evolve between $S R$ and $D M$. When $S R>D M$ (e.g. to the left of $I_{S}$ ), then the products of the stress responses dominate the products of the damage mechanisms by some type of steady-state process. The opposite is true on the other side of $I_{S}$ : damage products will dominate over those of the stress responses via some type of steady-state process. Thus, thermodynamic, not kinetic, factors determine the relative intensities of the $D M$ and $S R$ in the $\mathrm{P}$ and $\mathrm{D}$ domains.

In fact, we can say that, within the scope of our thinking, the kinetic-based death in $\mathrm{N}$ and the thermodynamic-based death in D are precisely the causes of cell death during ischemia or during reperfusion, respectively. We here only introduce the notion that our model provides an alternative view of cell death causality, and return to this critical point in the $3^{\text {rd }}$ paper of this series. 


\section{Summary}

To summarize, we have in this section explained how the sequence of phenotypes in the post-ischemic state space, $\mathrm{H}, \mathrm{P}, \mathrm{D}$ and $\mathrm{N}$, follow directly from the mutual antagonism between $S R$ and $D M$. With network thinking, we can understand the evolution of the phenotypes and make logical and systematic statements about ischemia-induced cell death. In fact we can state in precise terms the causes of ischemia-induced cell death.

This all follows with no reference whatsoever to the underlying details, a perhaps surprising situation to advocates of the traditional view that the causes of cell death are to be found somewhere in the fine details of the "ischemic cascade". On the other hand, the view presented here would be impossible to conceive without the detailed understanding of ischemia-induced damage and stress responses accumulated over the past few decades. As previously stated, the network view unifies and envelopes (encompasses) our present understanding of brain ischemia. It is a generalization and abstraction of decades of detailed results.

The final topic now to consider is how the $D M / S R$ competition may be modeled with a bistable circuit to produce the post-ischemic state space.

\section{Bistability and the post-ischemic state space}

To do this section justice, we must briefly discuss bistability and review additional details of how the differentiation examples discussed in the $1^{\text {st }}$ paper were modeled as bistable systems.

Bistability

Bistability is a common form of biological regulation and occurs whenever a system faces two mutually exclusive outcomes (Craciun et al 2006; Chatterjee et al 2008; Pomerening 2008). Examples of biological systems exhibiting bistable behavior include sonic hedgehog signal transduction (Lai et al 2004), determination of cell fate (discussed ahead; Huang et al 2007), microbial sporulation (Igoshin et al 2006), growth factor-induced endocytosis (Zwang and Yarden 2009), cell cycling in xenopus oocytes (Ingolia 2005), the alternation between REM and NREM sleep (Karlsson et al 2004), and apoptosis (Wee and Aguda 2006), among many others.

It is surprising that in these and other complex multidimensional systems, the control of outcome can be reduced to two mutually antagonistic variables. We continue with the example of differentiation started earlier to illustrate how in practice one models a system as bistable. In doing so, the logic of reducing the complex changes induced by brain ischemia to only two mutually antagonistic variables, $D M$ and $S R$, will be seen to be a maneuver that allows us to frame brain ischemia as another example of bistability in nature.

\section{GATA1/PU.1 Control of FDCP Cell Differentiation}

We briefly review the work of Huang et al (2007) on the differentiation of FDCP-mix (FDCP) progenitor cells into either erythroid cells or myeloid/monocytic cells. This is a bistable system in which the differentiation of FDCP cells into either erythroid or myeloid/ monocytic cells is a mutually exclusive decision.

The mutual exclusivity of the decision rests on eliciting different patterns of gene expression in the FDCP precursors. As is now well known, lineage specification is often controlled by "master" transcription factors (TFs) (Kim et al 2009). These are TFs that sit at the top of a hierarchy of gene control. Activation of the master TF induces downstream TFs that may 
induce further downstream TFs, where the final set of downstream TFs turns on the genes of the final differentiated phenotype (Boheler 2009).

The lineage fate decision of the FDCP cells is controlled by the mutual antagonism of two master TFs. GATA1 triggers erythroid differentiation and PU.1 triggers monocytic differentiation (Huang et al 2007). Overexpression of either TF by transfection will induce its respective phenotype (Graf 2002). Key to this whole system, GATA1 and PU.1 are mutually inhibitory. GATA1 suppresses PU.1 transcription, and vice versa (Huang et al 2007). Therefore, the entire complex set of hierarchical changes in the gene regulatory network of the FDCP cell can be reduced simply to the mutual antagonism between GATA1 and PU.1, allowing FDCP differentiation to be modeled as a bistable system.

We point out that when we discussed gene network state spaces in the $1^{\text {st }}$ paper these were described in terms of Boolean networks, and the input functions were binary. The following model of GATA1/PU.1 bistability is not binary but is modeled using continuous input functions where protein concentrations are continuous, not binary, variables (Alon 2006). Ahead, we emulate this approach and model the DM/SR competition also in terms of expressing these as continuous, not binary, variables.

\section{Modeling the GATA1/PU.1 Competition as a Bistable System}

Figure 7 illustrates the bistable models of the GATA1/PU.1 competition (Figure 7A and B) and of brain ischemia (Figure 7C). Images shown in Figures 7A and 7B are precise adaptations of data from Huang et al (2007). Here, the concentrations of GATA1 and PU.1 are notated as $x$ and $y$. Notating Figures 7A and 7B generically is meant to illustrate that the underlying equations are generic, and not dependent upon the specifics of the GATA1/PU.1 system. The underlying generic model is that of a bistable system. The GATA1/PU.1 system happens to fit this pattern. We argue in the next section that brain ischemia also fits this pattern. The circuit representations for each system are shown across the tops of the columns in Figure 7.

Figure 7A shows a pure bistable network that models only the mutual antagonism of $x$ and $y$. Under the circuit diagram is a cross section through the resulting state space. The points A and $\mathrm{B}$ are the attractors associated with high $[x]$ and high $[y]$, respectively. If $\mathrm{A}$ is the erythroid phenotype, then $x$ represents GATA1. Similarly, if B is the monocytic phenotype, then $y$ represents PU.1. One can see that if $[x]>[y]$ (e.g. [GATA1] $>$ [PU.1]), then the system is attracted to $A$, the erythroid phenotype, and vice versa if $[y]>[x]$. At the center of the cross section where $[x]=[y]$ is again a "watershed" point where the slope reverses sign. On either side of this "watershed" point, the system is fated to move to its respective attractor. Simply stated, in this pure bistable circuit, the TF with the higher concentration wins the competition and determines the outcome phenotype.

Underneath the cross section is a quasi-potential plot of the entire state space. In 3D, the state space in Figure 7A resembles a saddle (but it must be kept in mind that the state space itself is a two dimensional surface in $x$ and $y$ and the $3^{\text {rd }}$ dimension is a quasi-potential projection). The state space bends down to the attractors at $\mathrm{A}$ and $\mathrm{B}$, the lowest (e.g. most stable) points in the state space. The $x$ and $y$ axes extend from 0 to 1 , but the range is arbitrary and can simply be thought of as a normalized range. Note that A is the point $(1,0)$, the maximum of $x$ and the minimum of $y$, and vice versa for attractor B. All other points on the state space represent different proportions of $x$ and $y$ and correspond to all possible combinations of concentrations of the two TFs. All other points on the surface are unstable relative to the attractors (e.g. are at some higher point in the quasi-potential $3^{\text {rd }}$ dimension). The highest, most unstable points of the state space are at $(0,0)$ and $(1,1)$. The "watershed" point in the cross section is actually a point on the line (technically, geodesic, because the 
2D surface is bent) from $(0,0)$ to $(1,1)$. This line is called a separatrix; it is the unstable state between the two attractors in which the system is poised to go in either direction. The separatrix is designated on each state space in Figure 7D. We can now see why the term "ischemia on the separatrix", $I_{S}$, was introduced earlier. It refers to the point on the state space cross-section that lies on the separatrix of the 3D state space.

One of the objectives of Huang et al (2007) was to generate a state space that contained not only the final differentiated phenotypes (e.g. A and B), but also included the attractor for the undifferentiated FDCP cells. To do this, he modified the input functions as shown in Figure 7B. He added one additional feature about GATA1 and PU.1 to the bistable circuit: each activates its own transcription. This is now depicted in the circuit diagram by the rounded arrows emanating from and returning to $x$ and $y$ in Figure 7B. This modification adds positive feedback to each TF's control of its own transcription.

Adding positive feedback to the circuit modified the parameters of the input function equations (Huang et al 2007). The effect on the resulting state space is shown again in cross section and as a quasi-potential plot (Figure 7B). By adding the self-activation of each TF, the input functions now generated a third attractor in the state space, here labeled $\mathrm{C}$. The new attractor $\mathrm{C}$ represents the FDCP progenitor cell. It should be noted that the new attractor $\mathrm{C}$ fell along the separatrix of the pure bistable model, and generated a tristable system. However, attractor $\mathrm{C}$ is at a higher quasi-potential than either $\mathrm{A}$ or $\mathrm{B}$, thereby making it inherently less stable than A or B. In this fashion, Huang et al (2007) were successful in generating a realistic model of the phenotypes of the FDCP cell by simply adding only one biologically relevant fact to the pure GATA1/PU.1 bistable circuit: each TF activates its own transcription.

\section{Bistability and Brain Ischemia}

The above was necessary to set the background to discuss how the post-ischemic state space derives from a bistable competition between $D M$ and $S R$. The important take-home message is this: one can modify the input functions of the pure bistable circuit and alter the shape of the state space. Such modifications involve adding reasonable additional features to the core bistable antagonism of the two variables. We now discuss possible ways to modify the pure bistable circuit to get equations that give us back the post-ischemic state space as shown in Figures 3 and $7 \mathrm{C}$.

The post-ischemic state space is now depicted to mimic as closely as possible the results of Huang et al (2007) (Figure 7C). The images shown in Figure 7C constitute precisely the faux bistable network model of global brain ischemia. We can envision the end result. The issue is how to work backwards from the surface in Figure $7 \mathrm{C}$ to the input functions.

\section{Mapping Ischemia and Differentiation}

Let us first discuss the leaps in logic required to map the ischemia problem to the differentiation problem. This mapping occurs through a series of substitutions:

1. Where FDCP differentiation is control by two mutually antagonistic transcription factors, brain ischemia is controlled by two mutually antagonistic abstract variables, $D M$ and $S R$.

This may seem an unequal substitution in that GATA1 and PU.1 are physical, individual proteins, whereas $D M$ and $S R$ refer to total effects of many individual damage and stress responses, respectively, induced in neurons by ischemia. The issue here involves identifying two mutually antagonistic factors that control the system of interest. It happens to be physical proteins in the GATA1/PU.1 system. For brain ischemia, it happens to be the 
consequence associated with any specific ischemia-induced change. One is hard pressed not to assign a value of "good" or "bad" to any particular change identified in the post-ischemic brain. Or we could as well say "contributes to survival" and "contributes to death". If there is some other possible consequence of an ischemia-induced change, it escapes this author. If a change is "neutral" (with respect to outcome) then why consider it? There is the possibility that a specific change can flip state as a function of its position on the state space landscape. That is, a particular response could go from "good" $(S R)$ to "bad" $(D M)$ or vice versa. In such a fashion we could formally model Eng Lo's (2008) suggestion about the "biphasic" nature of stress responses. Here we simply point this out but do not discuss it further.

Therefore, we now see the main reason for classifying all changes induced in the brain as either contributing to survival (stress responses) or to cell death (damage mechanisms) and further, lumping these together in aggregate as the variables $D M$ or $S R$. Doing so provides two mutually antagonistic variables that allow us to model brain ischemia as a bistable system. In the $3^{\text {rd }}$ paper we address the relationship between the variables $D M$ and $S R$ and the many individual molecular changes of which each consists. At this point in the discussion, we justify this leap in logic by the simple fact that it seems to work.

2. Where the plots in Figure 7A/B are plots of protein concentrations ( $x$ and $y$ ), we substitute the intensities of the $D M$ and $S R$.

This does not seem unreasonable as concentrations are nonnegative continuous variables, and as Figure 6 indicates, the intensities of the $D M$ and $S R$ can so be depicted as well.

3. Where the changes in concentrations of $x$ and $y$ are a function of time in the input functions (e.g. $\frac{d[x]}{d t}$ and $\frac{d[y]}{d t}$ ) (Huang et al 2007), the changes in the $S R$ and $D M$ are a function of the amount of ischemia (e.g. $\frac{d S R}{d I}$ and $\frac{d D M}{d I}$; where $I$ is the amount of ischemia).

Even though we discussed the relationship between the amount of ischemia, $I$, and duration in the $1^{\text {st }}$ paper, the explicit role of time in the change of the intensities of the $S R$ and $D M$, considered as continuous variables, is obviously complicated. The discussion of kinetic and thermodynamic control would factor in here. It is likely that being able to formulate this question precisely in terms of rate and steady-state equilibrium expressions will allow derivation of the proper input functions for the post-ischemic state space. These would be precisely the functions $f$ and $g$ discussed earlier.

4. Where A and B are differentiated phenotypes, for brain ischemia these correspond to the normal neuron phenotype $\mathrm{S}$ and the cell death phenotype $\mathrm{CD}$. No caveats can be envisioned as to why substituting phenotypes would present a problem.

\section{Comparison of the Post-Ischemic and Differentiation State Spaces}

Let us next compare the bistable representation of the post-ischemic state space to those of differentiation. In Figure 7C, the cross section through the state space from point $\mathrm{S}$ to point $\mathrm{CD}$ captures all the features shown previously in Figure 3. Under the cross section, the intensities of the $D M$ and $S R$ are depicted as concentration (intensity) triangles by analogy to the Huang et al (2007) data. These however are inaccurate as depicted, thus the red question mark through them. The main inaccuracy is that they are shown as symmetric. From Figure 6 , it is clear that they should be asymmetric because, as discussed, the $S R$ and $D M$ do not both increase in the same manner as a function of the amount of ischemia, $I$. At present, the best that could be accomplished is to estimate input functions from the curves of $D M$ and $S R$ as shown in Figure 6; a chore for a future paper. 
The 3D post-ischemic state space representation has several features different from those of the differentiation state spaces (easily compared in panel D of Figure 7, where all three are aligned next to each other). First, the post-ischemic state space is clearly asymmetrical across the separatrix, whereas both differentiation state spaces are mirror images across the separatrix. The symmetry in the differentiation cases is due to symmetric parameters in the input functions (Huang et al 2007). Choice of asymmetric parameters would generate an asymmetric space, but these would simply be distorted mirror images across the separatrix. The asymmetry in the post-ischemic state space is of a more fundamental nature, being due to the $\mathrm{P}$ phenomena on the $\mathrm{S}$ but not the $\mathrm{CD}$ side of the separatrix. $\mathrm{P}$ simply does not mirror image itself functionally. Thus, we see the issue of the P-D boundary is nontrivial and reflects the intrinsic asymmetry of the post-ischemic state space.

The circuit diagram for the post-ischemic state space is shown with question marks because we do not yet know exactly how to modify the pure bistable circuit to generate the postischemic state space. For the GATA1/PU.1 bistable circuit, adding positive feedback altered the state space in Figure 7A by generating a shallow valley, the attractor C, in Figure 7B. We require the opposite change in the post-ischemic state space: a low flat hill to represent the $\mathrm{D}$ pheno-type. In addition the slopes associated with the $\mathrm{H}$ and $\mathrm{N}$ subregions of the state space are not expected to be mirror images.

Just to go through the intellectual exercise, if we, by analogy to the differentiation case in Figure 7B, add positive feedback to $D M$ and $S R$, that would mean that damage would beget damage and the stress responses would induce themselves. Neither of these is physically realistic. Generally, stress response pathways are the opposite, they are self limiting. As stress gene products accumulate, they inhibit their own transcription, as for example with the heat shock response (Balakrishnan and De Maio 2006). In addition, as we said, SR is expected to saturate with increasing $I$. And while some damage mechanisms are chain reactions (again, lipid peroxidation), in general damage does not increase in a positive feedback after ischemia has ended. No, it seems reasonable, as a first approximation, to assume that $D M$ increases as a linear function of $I\left(\right.$ after $\left.T_{P}\right)$, as depicted in Figure 6. So, while we know positive feedback is not the required modification to the pure bistable circuit, going through this exercise illustrates the type of thinking required to find the necessary modification(s).

As the above discussion illustrates, the bottle neck to getting input functions lies precisely in answering the question: how is the pure bistable circuit to be modified to alter the shape of the state space to match that of our post-ischemic state space that we have derived based on the mass of empirical evidence? A first stab at an answer is that addition of saturation to the function $S R=g(I)$, and leaving the function $D M=f(I)$ linear with respect to $I$ (e.g. when $I$ > $T_{P}$ ) may generate the shape we have here hypothesized for the post-ischemic state space. There are also related empirical questions:

1. What are the real graphs (or some reasonable approximation) of the relative intensities of the $S R$ and $D M$ as a function of the amount of ischemia, I? What molecular markers could provide this information?

2. 2. Is the P-D boundary (e.g. at $T_{D}$ ) a continuum or is there a wall (quasi-potential barrier) there (Figure 5)? Again we raise this point because $P$ introduces an intrinsic asymmetry to the state space. This is not a question to be answered by theory. It needs to be answered empirically by a demonstration (or not) that resistant neurons in $\mathrm{D}$ are in fact preconditioned. To our knowledge, this issue has not been considered before, but the issue is clearly experimentally accessible, and answering it will provide an important clue to the shape of the post-ischemic state space. 


\section{Open ends}

On one hand it is perhaps unsatisfying that this story does not yet have a closed ending, and that we instead end this discussion with our sleeves rolled up considering technical issues. This is science after all, it is what we do, and many hands make light work.

On the other hand, we have here the beginnings of a viable reformulation of what brain ischemia is. The core of this reformulation is to model brain ischemia as a bistable system in a network context. Again, and quite obviously, post-ischemic neurons face the mutually exclusive decision to either live or die. Recognizing that bistability underlies brain ischemia brings it into the distinguished company of a myriad other biological processes that have bistability at the core of their operation.

Clearly the approach described here is not simply a metaphor or vague insight. It provides a new means to conceptualize, systematize and mathematically model brain ischemia. It is an alternative to the "ischemic cascade" as an explanatory framework. It does not reject the "ischemic cascade"; it encompasses it in a new context where the myriad details of the "ischemic cascade" become subsumed as the points of the state space (more said on this in the $3^{\text {rd }}$ paper).

This "Brain Ischemia 2.0" offers new insights into the causes of cell death by introducing the kinetic and thermodynamic explanations completely independent of any specific physiologic or molecular detail. It can unify our conception of brain ischemia by bringing all the ischemia-induced phenomena within the scope of one mathematical framework. Finally, the model hints at a general theory of cell injury. The logic used here could be applied to other brain injuries (and in fact has, Villoslada et al 2009), other cell types, other organs. While the sky is the limit in this regard, as the above makes plain, we still have to complete the project of modeling brain ischemia before moving on to other applications. But even at its present stage of development, the Brain Ischemia 2.0 viewpoint offers a fresh look at the current crisis in therapeutics, which we discuss in the next, the $3^{\text {rd }}$ paper of this series.

\section{Acknowledgments}

The full acknowledgements are presented in the $4^{\text {th }}$ paper of this series. This work was supported by the National Institute of Neurological Disorders and Stroke (NS-057167).

\section{Abbreviations}

$\begin{array}{ll}\text { 3D } & \text { three dimensional } \\ \text { CBF } & \text { cerebral blood flow } \\ \text { CD } & \text { the attractor for cell death } \\ \text { CGI } & \text { complete global ischemia } \\ \text { D } & \text { delayed neuronal death region of post-ischemic state space } \\ \text { DM } & \text { effective total ischemia-induced damage } \\ \text { DND } & \text { delayed neuronal death } \\ \text { H } & \text { homeostatic region of post-ischemic state space } \\ \text { I } & \text { the amount of ischemia } \\ \text { IS } & \text { the amount of ischemia at the separatrix } \\ \text { N } & \text { necrotic region of post-ischemic state space }\end{array}$


$\mathbf{P} \quad$ preconditioning region of post-ischemic state space

S the attractor for the steady-state pheno-type of a neuron

SR effective total ischemia-induced stress response capacity

$\mathbf{T}_{\mathbf{D}} \quad$ threshold of delayed neuronal death

TF transcription factor

$\mathbf{T}_{\mathbf{N}} \quad$ threshold of necrosis

$\mathbf{T}_{\mathbf{P}} \quad$ threshold of preconditioning

3D three dimensional

CBF cerebral blood flow

\section{References}

Alon, U. An Introduction to Systems Biology: Design Principles of Biological Circuits. Chapman \& Hall/Crc; London, UK: 2006.

Balakrishnan K, De Maio A. Heat shock protein 70 binds its own messenger ribonucleic acid as part of a gene expression self-limiting mechanism. Cell Stress Chaperones 2006;11:44-50. [PubMed: 16572728]

Boheler KR. Stem cell pluripotency: a cellular trait that depends on transcription factors, chromatin state and a checkpoint deficient cell cycle. J Cell Physiol 2009;221:10-17. [PubMed: 19562686]

Chan PH. Oxygen radicals in focal cerebral ischemia. Brain Pathol 1994;4:59-65. [PubMed: 8025703]

Chatterjee A, Kaznessis YN, Hu W-S. Tweaking biological switches through a better understanding of bistability behavior. Current Opinion in Biotechnology 2008;19:475-481. [PubMed: 18804166]

Craciun G, Tang Y, Feinberg M. Understanding bistability in complex enzyme-driven reaction networks. Proc Natl Acad Sci USA 2006;103:8697-8702. [PubMed: 16735474]

Graf T. Differentiation plasticity of hematopoietic cells. Blood 2002;99:3089-3101. [PubMed: 11964270]

Huang S, Guo YP, May G, Enver T. Bifurcation dynamics in lineage-commitment in bipotent progenitor cells. Dev Biol 2007;305:695-713. [PubMed: 17412320]

Huang S, Ingber DE. Shape-dependent control of cell growth, differentiation, and apoptosis: switching between attractors in cell regulatory networks. Exp Cell Res 2000;261:91-103. [PubMed: 11082279]

Huang S. Reprogramming cell fates: reconciling rarity with robustness. BioEssays 2009;31:546-560. [PubMed: 19319911]

Iadecola C, Nedergaard M. Glial regulation of the cerebral microvasculature. Nat Neurosci 2007;10:1369-1376. [PubMed: 17965657]

Igoshin OA, Price CW, Savageau MA. Signaling network with a bistable hysteretic switch controls developmental activation of the sigma transcription factor in Bacillus subtilis. Mol Microbiol 2006;61(1):165-184. [PubMed: 16824103]

Ingolia N. Cell cycle: bistability is needed for robust cycling. Curr Biol 2005;15:R961-963. [PubMed: 16332526]

Karlsson KA, Kreider JC, Blumberg MS. Hypothalamic contribution to sleep-wake cycle development. Neuroscience 2004;123:575-582. [PubMed: 14698764]

Kim HD, Shay T, O'Shea EK, Regev A. Transcriptional regulatory circuits: predicting numbers from alphabets. Science 2009;325:429-432. [PubMed: 19628860]

Kirino T. Delayed neuronal death. Neuropathology 2000;20(Suppl):S95-97. [PubMed: 11037198]

Kirino T. Ischemic tolerance. J Cereb Blood Flow Metab 2002;22:1283-1296. [PubMed: 12439285]

Lai K, Robertson MJ, Schaffer DV. The sonic hedgehog signaling system as a bistable genetic switch. Biophys J 2004;86:2748-2757. [PubMed: 15111393] 
Lo EH. A new penumbra: transitioning from injury into repair after stroke. Nat Med 2008;14:497-500. [PubMed: 18463660]

Mendel G. Versuche über Pflanzen-Hybriden. Verh Naturforsch Ver Brünn 1866;4:3-47. (in English in 1901, J R Hortic Soc 26:1-32).

Mrsulja BB, Ueki Y, Lust WD. Regional metabolite profiles in early stages of global ischemia in the gerbil. Metab Brain Dis 1986;1:205-220. [PubMed: 3508242]

Pomerening JR. Uncovering mechanisms of bistability in biological systems. Curr Opin Biotechnol 2008;19:381-388. [PubMed: 18634875]

Safar P. Cerebral resuscitation after cardiac arrest: a review. Circulation 1986;74:IV138-153. [PubMed: 3536160]

Siesjö BK, Smith ML. The biochemical basis of ischemic brain lesions. Arzneimittelforschung 1991;41:288-292. [PubMed: 1859497]

Siesjö BK, Wieloch T. Cerebral metabolism in ischaemia: neurochemical basis for therapy. Br J Anaesth 1985;57:47-62. [PubMed: 3917676]

Sykes, P. A Guidebook to Mechanism in Organic Chemistry. 6th Edition. Pearson Prentice Hall; 1986. 1986.

Traystman RJ, Kirsch JR, Koehler RC. Oxygen radical mechanisms of brain injury following ischemia and reperfusion. J Appl Physiol 1991;71:1185-1195. [PubMed: 1757340]

Villoslada P, Steinman L, Baranzini SE. Systems biology and its application to the understanding of neurological diseases. Ann Neurol 2009;65:124-139. [PubMed: 19260029]

Waddington, $\mathrm{CH}$. The strategy of the genes. Allen and Unwin; London: 1957.

Wee KB, Aguda BD. Akt versus p53 in a network of oncogenes and tumor suppressor genes regulating cell survival and death. Biophys J 2006;91:857-865. [PubMed: 16648169]

White BC, Sullivan JM, DeGracia DJ, O'Neil BJ, Neumar RW, Grossman LI, Rafols JA, Krause GS. Brain ischemia and reperfusion: molecular mechanisms of neuronal injury. J Neurol Sci 2000;179(S 1-2):1-33. [PubMed: 11054482]

Zwang Y, Yarden Y. Systems biology of growth factor-induced receptor endocytosis. Traffic 2009;10:349-363. [PubMed: 19183301] 
QIIJ Physiological range of homeostatic variation

Supra-physiological range of homeostatic variation

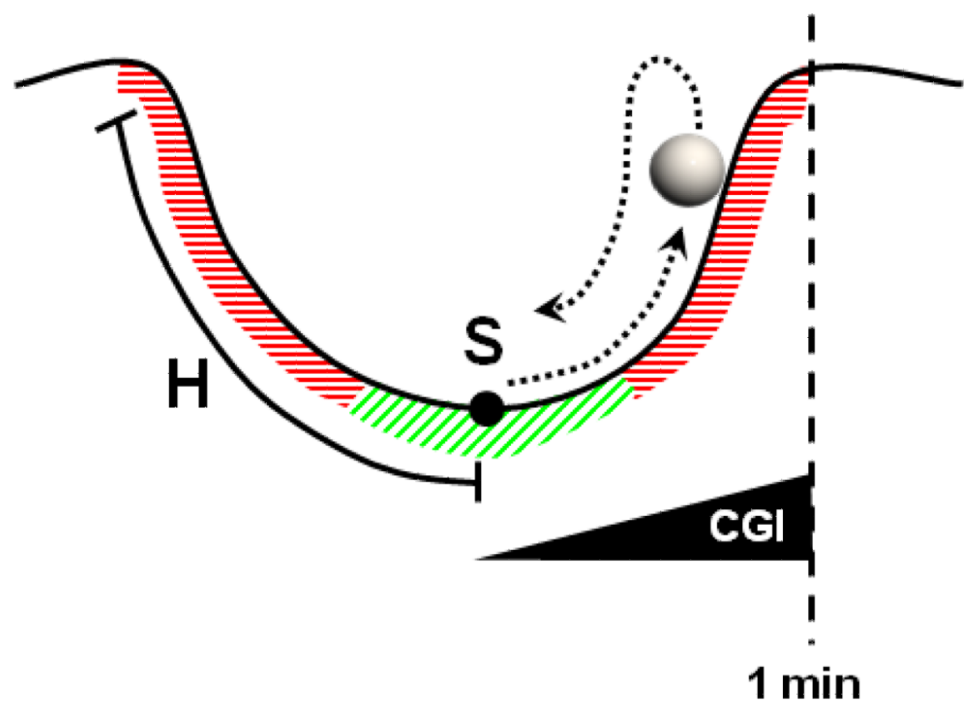

Figure 1.

The relationship between the steady-state network configuration of a neuron, $\mathrm{S}$, and homeostatic displacement into its basin of attraction, H. Here, we show a cross-section through the "valley", the attractor $\mathrm{S}$ and its basin of attraction, here called $\mathrm{H}$, of the neuron cell type in the gene network state space of an organism. The green range of $\mathrm{H}$ reflects physiological homeostatic responses; the red range represents supra-physiological responses. The steep slope of the supra-physiological (red) range of homeostatic variation is meant to represent the relative rapidity with which a neurons will return to steady-state, $\mathrm{S}$, following brief application of a small amount of complete global ischemia (CGI). The black triangle represents the amount of CGI associated with this response in neurons. Note the CGI triangle in this and in subsequent figures is not meant to be exact, but approximate insofar as exact values would require defining the species and method of inducing CGI. The marble represents the network configuration and its movement represents the trajectory of the configuration in state space. 


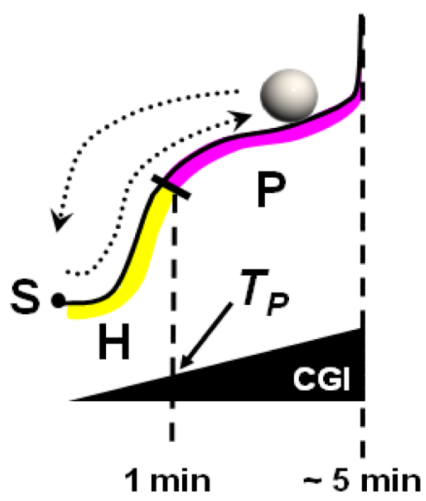

Figure 2.

Passing a threshold amount of ischemia, $T_{P}$, causes the neurons to shift into a new phenotype, generally called preconditioned. The hallmark of this phenotype is enhanced protection if a potentially lethal stimulus ensues while the phenotype persists. Again, a cross section is shown through the neuronal state space, this time from the middle of the $S$ attractor valley and moving outwards in one direction. The steady state configuration, $\mathrm{S}$, is shown at the bottom of the basin of attraction, H. A new plateau represents the preconditioned phenotype. This plateau extends for an amount of ischemia between the minimum and maximum amounts that will induce the preconditioned phenotype. The marble represents the neuronal network configuration. The trajectory (dotted arrows) illustrates both the onset and decay of the preconditioned phenotype. Because of its reversibility, this phenotype is called meta-stable. 


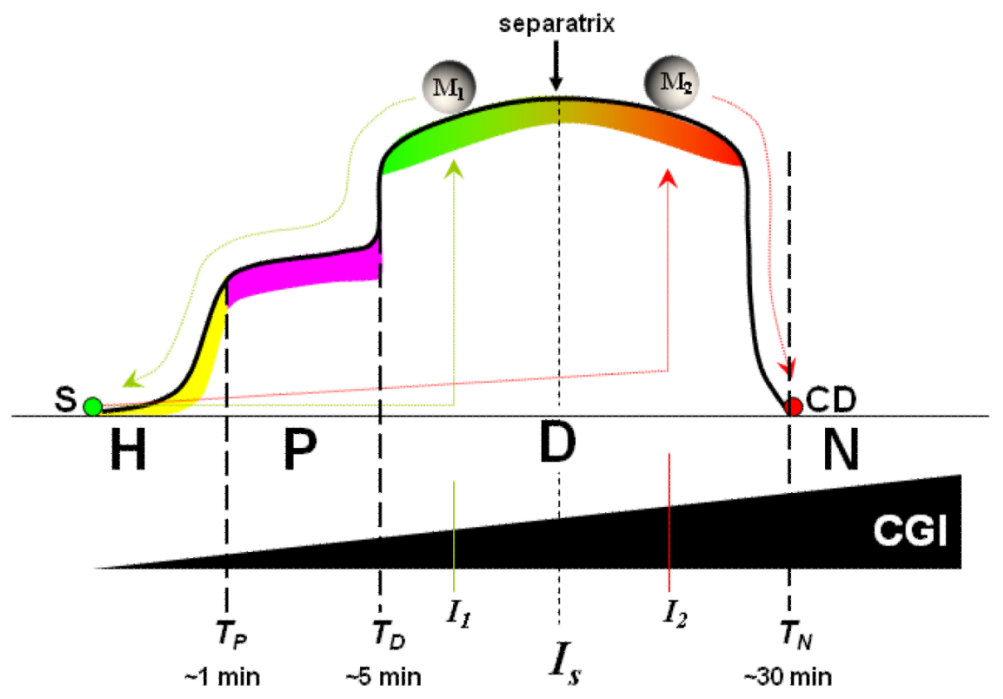

Figure 3.

A cross section through the expected state space of a post-ischemic neuron. Two attractors are depicted: the steady-state attractor of a normal neuron (green point, S) and the attractor for cell death (red point, CD). Cell death induced during ischemia occurs if the cell experiences amounts of ischemia $\geq T_{N}$. Between the thresholds $T_{D}$ and $T_{N}$, is the range of delayed neuronal death, D. This range possesses a "watershed" or tipping point at an amount of ischemia here labeled "ischemia at the separatrix" $\left(I_{S}\right)$ that separates the bistable outcome of survival from death. A resistant neuron (depicted by marble $\mathrm{M}_{1}$ ) will experience $I_{1}<I_{S}$ (green range of $\mathrm{D}$ ), follow the trajectory back to $\mathrm{S}$ and survive the ischemia. An ischemiavulnerable neuron will experience $I_{2}>I_{S}$ (red range of D), follow a trajectory to the attractor $\mathrm{CD}$ and die. The state space captures the fact that, depending upon the amount of ischemia, I, (here labeled "CGI", complete global ischemia) the cell death can occur after the ischemia (during reperfusion) in the $\mathrm{D}$ phenotype, or during the ischemia itself, in the $\mathrm{N}$ phenotype. $\mathrm{H}$ is shown by the yellow range and $\mathrm{P}$ by the purple range. The amounts of CGI shown as duration are only approximate and not meant to be exact. 

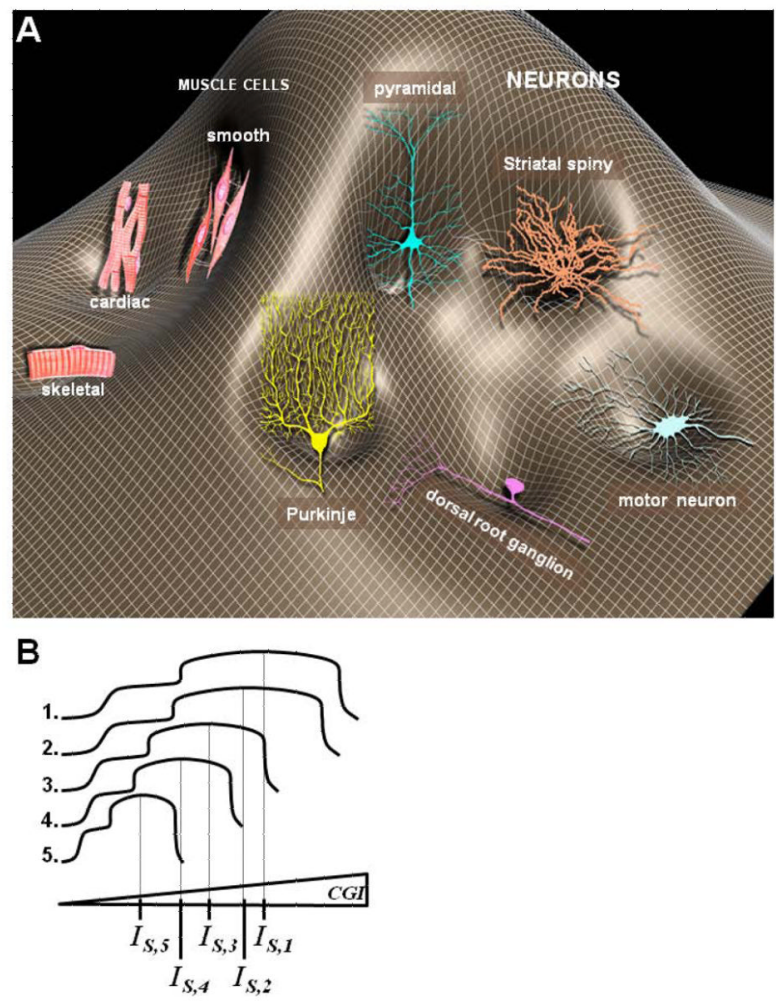

Figure 4.

(A) "Valley of the Neurons". The genomic state space for neurons is expected to make a broad valley within which all specific neuron subtype transcriptomes will occupy their own local attractor states in this "valley of the neurons". Shown here are a few of the vertebrate neuron types; the actual state space would contain the dozens of neuron subtypes typical of a vertebrate nervous system. Other cell types would inhabit their own territories on the landscape. The valley of muscle cells is shown here, another vertebrate cell type whose function is intimately linked to electrical conduction. (B). Different classes of neurons (simply labeled 1-5) will each possess their own state spaces. It is assumed that the form of the state spaces will be generally similar in terms of the post-ischemic phenotypes, but that the thresholds will differ amongst neuron subtypes, consistent with the well-established differential ischemic vulnerability of neuronal classes. The five hypothetical neuron postischemic state spaces are shown in order of decreasing resistance to ischemia, as indicated by the decreasing values of $I_{S}$ for each neuron type. 


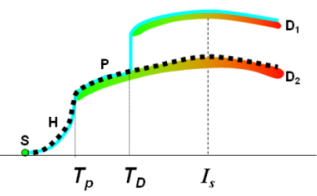

Figure 5.

Two alternate ways to conceive of the relationship between the $\mathrm{P}$ and $\mathrm{D}$ post-ischemic phenotypes. The cyan line recapitulates that shown in Figure 3 where P and D $\left(D_{1}\right.$ above) are distinguishable phenotypes because a barrier is presupposed at the $T_{D}$ threshold. The alternative is shown by the dotted line in which the $\mathrm{P}$ and $\mathrm{D}\left(\mathrm{D}_{2}\right)$ phenotypes are continuous. Both cases would give the same net post-ischemic outcome, but each would imply different underlying equations. 


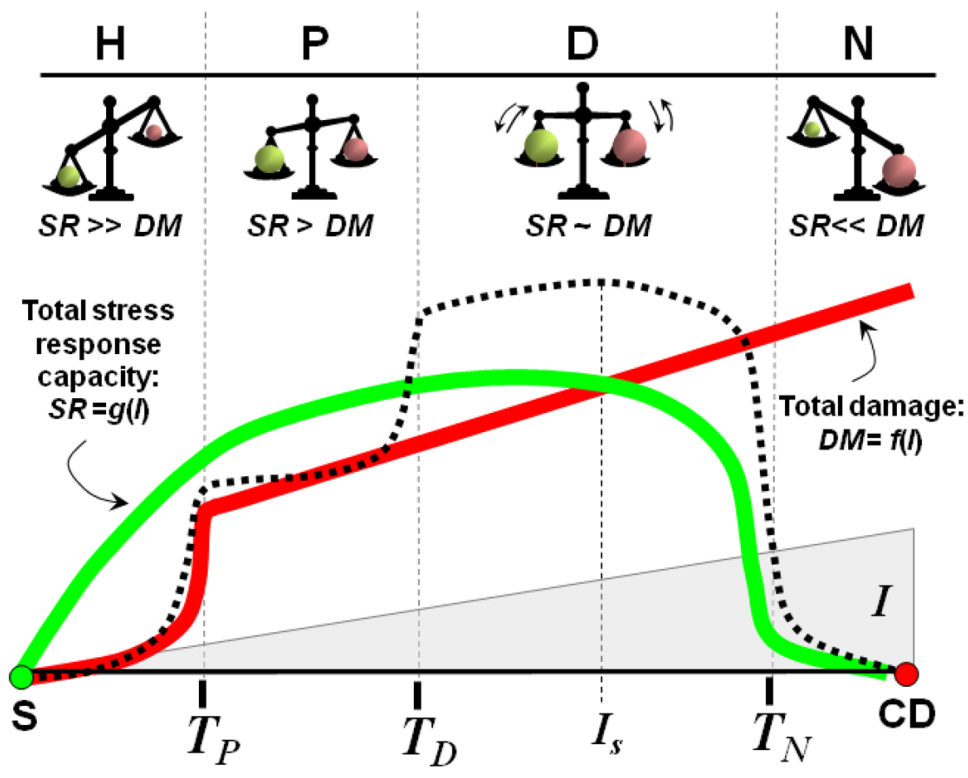

Figure 6.

The shape of the post-ischemic state space follows from the competition between the total effective damage $(D M)$ and the total stress response capacity $(S R)$ induced in neurons by ischemia. Along the top, the relative intensities of $D M$ and $S R$ are depicted as different sized balls weighed on scales. Below, the green and red curves represent the relative intensities of the $D M$ and $S R$, respectively, as functions $f(I)$ and $g(I)$, where $I$ is the amount of ischemia. Milestone events are associated with each of the ischemic thresholds (see text for details). The black dotted line is an outline of the cross section through the post-ischemic neuronal state space as shown in Figure 3. The sequence of H, P, D and N can be seen here to naturally follow from the mutual antagonism of the $S R$ and $D M$. When $S R>D M$, the system naturally falls back to the steady-state attractor of the neuron, $\mathrm{S}$ (green dot). When $D M>S R$, then the system falls to the cell death attractor CD (red dot). $S R=D M$ at $I_{S}$, the amount of ischemia at the separatrix, which is the tipping point between survival and death. 


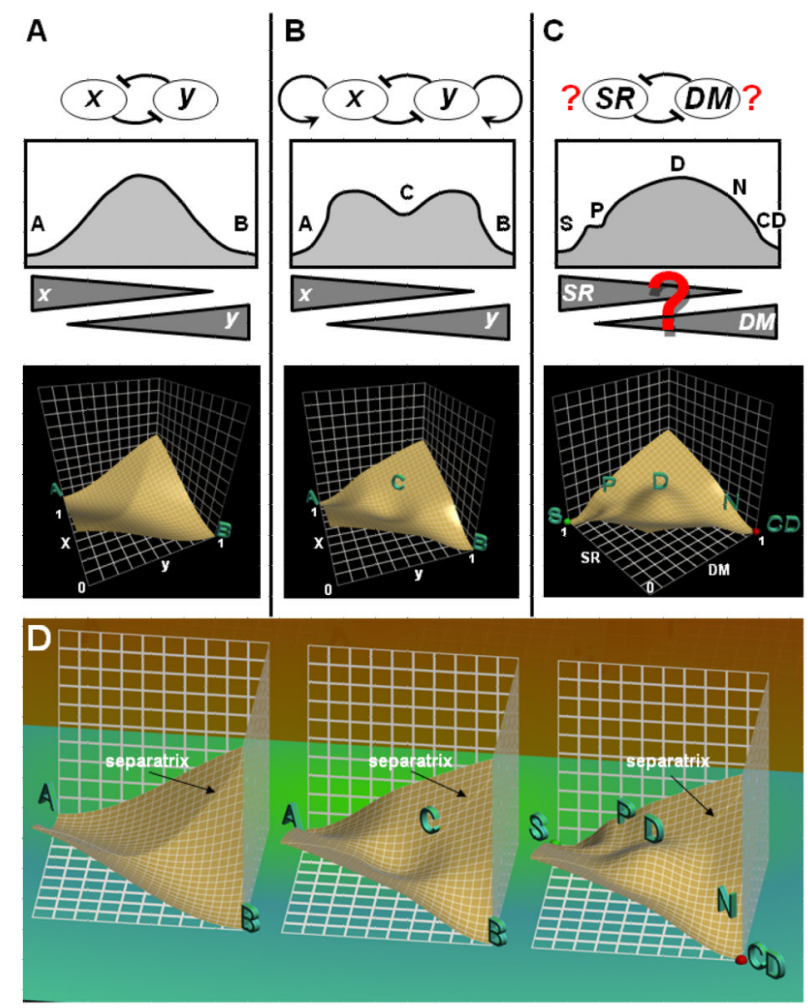

Figure 7.

Bistable models of differentiation (A and $\mathbf{B}$ ) and brain ischemia $(\mathbf{C})$. Circuit diagrams are shown at the top of each column. Under these are shown cross sections through the state spaces that derive from the equations of the respective circuit diagrams. Below the cross sections are quasi-potential plots of the entire state space. (A) The pure bistable circuit produces a saddle-shaped state space, and generates only two attractors A and B, controlled by the levels of the variables $x$ and $y$, respectively. If $x>y$, the system is attracted to A, and vice versa if $y>x$. (B) Addition of positive feedback to $x$ and $y$ alters the bistable circuit and changes the shape of the resulting state space, producing a third attractor, C. C is at a higher quasi-potential, than attractors A and B. (C) Modifications to the bistable circuit to produce the post-ischemic state space are currently unknown and depicted as red question marks in the circuit diagram. In the cross sectional plot, the relative levels of $S R$ and $D M$ are also unknown as again marked by the red question mark. The relative levels of $S R$ and $D M$ are expected to follow the form shown in Figure 6. The quasi-potential state space plot of the post-ischemic state space shows the H, P, D and N phenotypes are controlled by the variables $S R$ and $D M$. The state of maximum $S R$ and minimum $D M$ corresponds to the attractor $\mathrm{S}$, and the state of minimum $S R$ and maximum $D M$ corresponds to the attractor CD. Changes in $S R$ and $D M$ occur as a function of the amount of ischemia, which would be the dependent variable of the input function equations. Bottom panel: The quasi-potential state spaces for the pure bistable, positive feedback-modified bistable, and post-ischemic system are shown next to each other for easy comparison. Note that a label for $\mathrm{H}$ is omitted from the post-ischemic state space simply to keep the images from becoming cluttered. The basin within which $\mathrm{S}$ is the minimum point is in fact $\mathrm{H}$. The separatrix is indicated in each state space by the dashed line extending from $(0,0)$ to $(1,1)$. 